\title{
Rivaroxaban vs. Warfarin in Japanese Patients With Non-Valvular Atrial Fibrillation in Relation to Age
}

\author{
- Insight From J-ROCKET AF -
}

\author{
Masatsugu Hori, MD, PhD; Masayasu Matsumoto, MD, PhD; Norio Tanahashi, MD; \\ Shin-ichi Momomura, MD; Shinichiro Uchiyama, MD, PhD; Shinya Goto, MD, PhD; \\ Tohru Izumi, MD, PhD; Yukihiro Koretsune, MD, PhD; Mariko Kajikawa, MD, PhD; \\ Masaharu Kato; Hitoshi Ueda, PhD; Kazuma Iekushi, MD, PhD; Satoshi Yamanaka, MD, PhD; \\ Masahiro Tajiri, BSc on behalf of the J-ROCKET AF Study Investigators
}

\begin{abstract}
Background: The J-ROCKET AF study found that rivaroxaban was non-inferior to warfarin with respect to the principal safety outcome in patients with atrial fibrillation (AF). The aim of this subgroup analysis was to assess the safety and efficacy of rivaroxaban and warfarin in relation to patient age.

Methods and Results: A total of $39.0 \%$ were elderly (aged $\geq 75$ years). In elderly patients, the principal safety outcome occurred at $25.05 \%$ /year with rivaroxaban vs. $16.95 \% / y e a r$ on warfarin (hazard ratio [HR], $1.49 ; 95 \%$ confidence interval $[\mathrm{Cl}]:$ 1.02-2.16), whereas the primary efficacy endpoint occurred at $2.18 \% /$ year vs. $4.25 \% /$ year (HR, $0.51 ; 95 \% \mathrm{Cl}: 0.20-1.27)$, respectively. There were significant interactions in the principal safety outcomes of rivaroxaban compared with warfarin between the elderly and non-elderly groups, but not in the primary efficacy endpoints $(\mathrm{P}=0.04$ and 0.82 for both interactions, respectively). Furthermore, in elderly patients, in the rivaroxaban group there was a trend to increase the principal safety outcome regardless of renal function. In elderly patients with preserved renal function, however, patients on rivaroxaban had a marginally favorable trend in the primary efficacy endpoint incidence rate compared with patients on warfarin.
\end{abstract}

Conclusions: There is a need to carefully consider the risks and benefits of therapy with rivaroxaban in elderly patients with non-valvular AF. (Circ J 2014; 78: 1349-1356)

Key Words: Atrial fibrillation; Elderly; Oral anticoagulation; Stroke prevention

$\mathbf{T}$ he number of patients with atrial fibrillation (AF) continues to increase, and $\mathrm{AF}$ is a very common cardiac arrhythmia with a prevalence of approximately $30 \%$ in the population aged $\geq 80$ years. ${ }^{1}$ The presence of $\mathrm{AF}$ independently increases the risk of mortality and morbidity with stroke, thromboembolism, congestive heart failure, and impaired quality of life, resulting in expanded health-care concerns. ${ }^{2}$ Anticoagulant therapy for stroke prophylaxis is considered essential for patients with AF, but this therapy is underused and poorly controlled, partly because of the concern of bleeding risk, particularly in elderly patients. ${ }^{3}$ Older age, however, is a component of not only the $\mathrm{CHADS}_{2}$ score for stroke risk, ${ }^{4}$ but also the HAS-BLED score for bleeding risk. ${ }^{5}$ Therefore, we need to refine the strategy that maximizes the benefits of anticoagulant therapy for stroke prevention while minimizing the risks of major hemorrhage for each individual patient.

Until recently, warfarin was the only clinically available oral anticoagulant that could effectively reduce the risk of stroke and systemic embolism in patients with AF. In recent years, however, rivaroxaban has been launched as a new oral anticoagulant. Rivaroxaban can inhibit thrombosis by the direct inhibition of Factor Xa via a mechanism of action that differs

Received November 6, 2013; revised manuscript received February 19, 2014; accepted February 20, 2014; released online April 7, 2014 Time for primary review: 14 days

Osaka Medical Center for Cancer and Cardiovascular Diseases, Osaka (M.H.); Institute for Clinical Research, Osaka National Hospital, Osaka (Y.K.); Bayer Yakuhin, Osaka (M. Kajikawa, M. Kato, H.U., K.I., S.Y., M.T.); Department of Clinical Neuroscience and Therapeutics, Hiroshima University, Hiroshima (M.M.); Department of Neurology, Saitama Medical University International Medical Center, Saitama (N.T.); Division of Cardiovascular Medicine, Saitama Medical Center, Jichi Medical University, Saitama (S.M.); Department of Neurology, Tokyo Women's Medical University, Tokyo (S.U.); Department of Medicine (Cardiology), Tokai University School of Medicine, Tokyo (S.G.); and Department of Cardio-angiology, Kitasato University School of Medicine, Sagamihara (T.I.), Japan

Mailing address: Masatsugu Hori, MD, PhD, Osaka Medical Center for Cancer and Cardiovascular Diseases, 1-3-3 Nakamichi, Higashinariku, Osaka 537-8511, Japan. E-mail: hori-ma@mc.pref.osaka.jp

ISSN-1346-9843 doi:10.1253/circj.CJ-13-1324

All rights are reserved to the Japanese Circulation Society. For permissions, please e-mail: cj@j-circ.or.jp 
Table. Baseline Patient Characteristics

\section{Characteristic}

Mean age (years)

Mean body weight $(\mathrm{kg})$

$\mathrm{CrCl}<50 \mathrm{ml} / \mathrm{min}(\%)$

Mean $\mathrm{CHADS}_{2}$ score

CHF (\%)

Hypertension (\%)

Diabetes mellitus (\%)

Prior stroke/TIA or systemic embolism (\%)
Female (\%)

\begin{tabular}{ccc}
\multicolumn{3}{c}{ Age $<75$ years } \\
\hline $\begin{array}{c}\text { Rivaroxaban } \\
(\mathbf{n}=\mathbf{3 8 7})\end{array}$ & $\begin{array}{c}\text { Warfarin } \\
(\mathbf{n}=\mathbf{3 9 3})\end{array}$ & P-value \\
66 & 66 & 0.29 \\
14.5 & 14.5 & 0.99 \\
66.7 & 67.5 & 0.34 \\
10.1 & 7.9 & 0.28 \\
3.0 & 3.0 & 0.74 \\
37.7 & 34.9 & 0.41 \\
77.5 & 77.9 & 0.90 \\
42.1 & 43.3 & 0.75 \\
71.6 & 71.2 & 0.92
\end{tabular}

\begin{tabular}{ccc}
\multicolumn{3}{c}{ Age $\geq \mathbf{7 5}$ years } \\
\hline $\begin{array}{c}\text { Rivaroxaban } \\
(\mathbf{n}=\mathbf{2 5 2})\end{array}$ & $\begin{array}{c}\text { Warfarin } \\
(\mathbf{n}=\mathbf{2 4 6})\end{array}$ & P-value \\
79 & 79 & 0.84 \\
21.0 & 33.3 & $<0.01$ \\
60.7 & 58.7 & $<0.05$ \\
40.5 & 45.5 & 0.25 \\
3.7 & 3.6 & 0.33 \\
46.8 & 48.8 & 0.66 \\
82.5 & 82.1 & 0.91 \\
34.1 & 27.2 & 0.10 \\
52.0 & 50.8 & 0.79
\end{tabular}

$\mathrm{CHADS}_{2}$, clinical prediction rule for estimating the risk of stroke for patients with atrial fibrillation, it was calculated by assigning 1 point for diagnoses of congestive heart failure, hypertension, age 75 years or older, and diabetes mellitus and 2 points for prior stroke or transient ischemic attack; $\mathrm{CHF}$, congestive heart failure; $\mathrm{CrCl}$, creatinine clearance; TIA, transient ischemic attack.

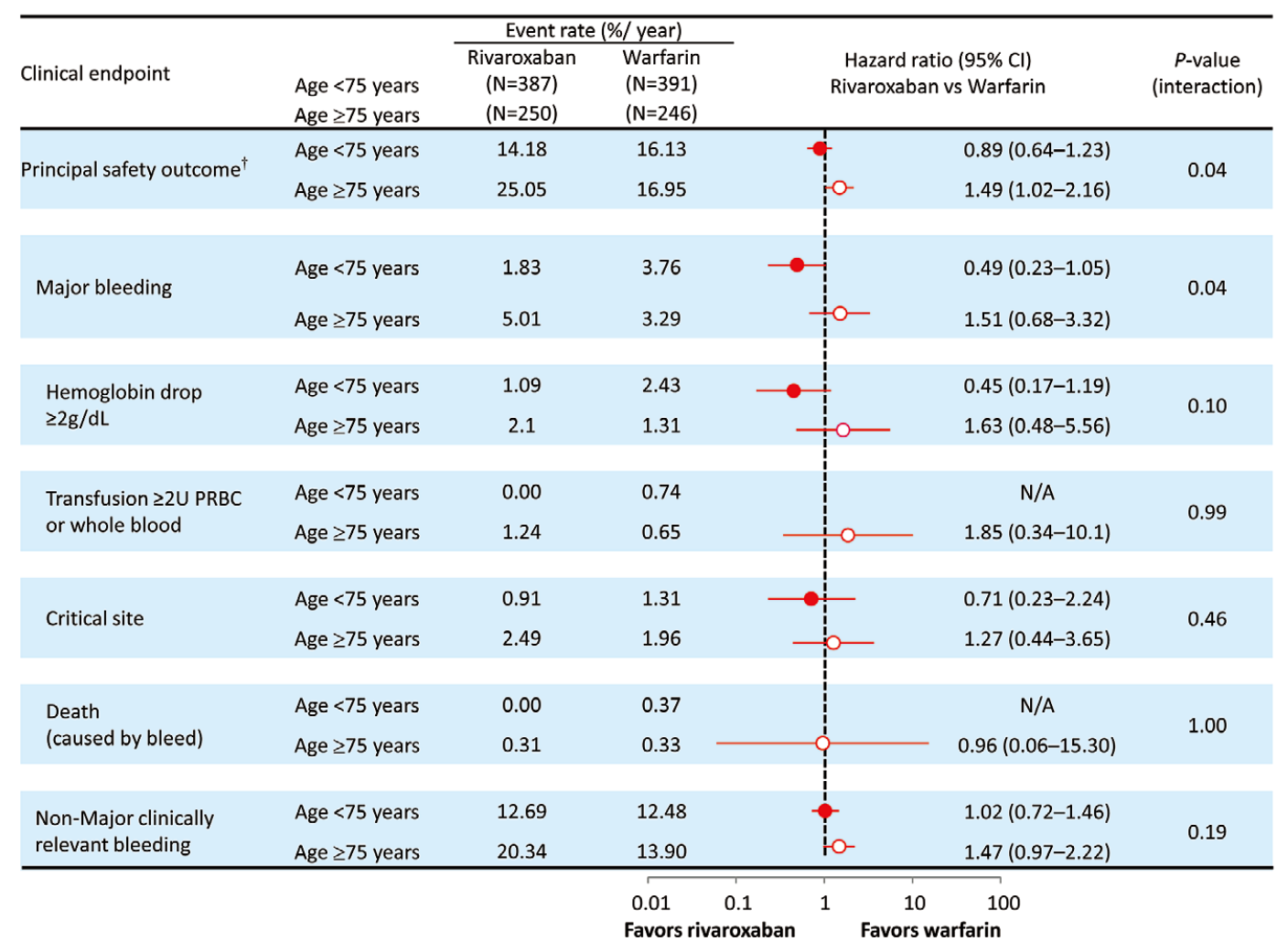

Figure 1. Principal safety outcomes in the safety group on treatment analysis according to age ( $\geq 75$ years or younger). Safety group, on treatment. ${ }^{\dagger}$ Major or non-major clinically relevant bleeding. $\mathrm{Cl}$, confidence interval; N/A, not applicable.

from that of warfarin. ${ }^{6-8}$ In J-ROCKET AF, the safety and efficacy of rivaroxaban were compared with those of dose-adjusted warfarin in accordance with the Japanese AF guidelines from the Japanese Circulation Society for Japanese patients with $\mathrm{AF}$ (a target international normalized ratio [INR] range of 1.6-2.6 for patients aged $\geq 70$ years, or 2.0-3.0 for those aged $<70$ years). ${ }^{9} \mathrm{~J}$-ROCKET AF targeted patients with a mean age of 71.1 years, with moderate or higher risk for thromboembolism and a $\mathrm{CHADS}_{2}$ score of $\geq 2$. In the present subgroup analy- sis, we investigated the safety and efficacy of rivaroxaban vs. dose-adjusted warfarin in elderly patients (aged $\geq 75$ years) or non-elderly patients (aged $<75$ years) with non-valvular AF in J-ROCKET AF.

\section{Methods}

Study Design, Participants, and Procedure

The design and results of J-ROCKET AF have been described 


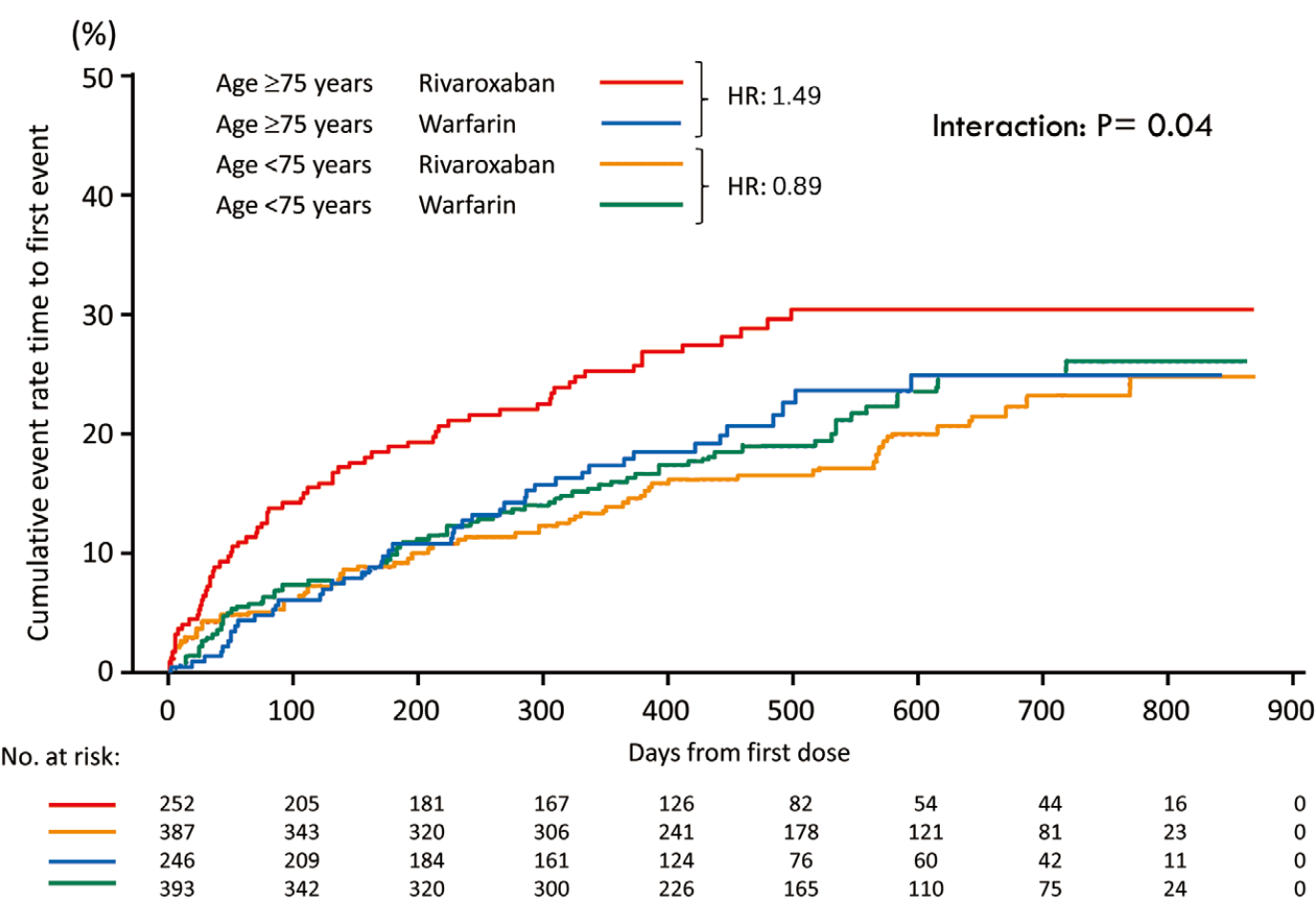

Figure 2. Kaplan-Meier curve of time to first major or non-major clinically relevant bleeding event according to age ( $\geq 75$ years or younger). Safety group, on treatment. HR, hazard ratio.

previously. ${ }^{9}$ In brief, J-ROCKET AF was a prospective, randomized, double-blind, double-dummy, parallel-group, activecontrolled, multicenter clinical trial comparing the safety of rivaroxaban vs. dose-adjusted warfarin in accordance with the Japanese guidelines in patients with non-valvular AF. The study was approved by the institutional review boards, and all patients gave informed consent. The trial was conducted in accordance with Japanese Good Clinical Practice. Japanese patients aged $\geq 20$ years with non-valvular AF, documented electrocardiographically $\leq 30$ days before enrollment, were randomized at 167 participating facilities in Japan. Patients had a history of prior ischemic stroke, transient ischemic attack, or non-central nervous system (CNS) systemic embolism, or had a CHADS 2 score $\geq 2$ based on the following risk factors for thromboembolism: congestive heart failure and/or left ventricular ejection fraction $\leq 35 \%$, hypertension (defined as use of anti-hypertensive medications within 6 months before the screening visit or persistent systolic blood pressure $>140 \mathrm{mmHg}$ or diastolic blood pressure $>90 \mathrm{mmHg}$ ), age $\geq 75$ years, or diabetes mellitus (ie, history of type 1 or type 2 diabetes mellitus or use of anti-diabetic medications within 6 months before the screening visit). Randomization of patients without prior stroke, transient ischemic attack, or non-CNS systemic embolism and with only 2 stroke risk factors limited the patient number to $<10 \%$ of those enrolled. Patients with creatinine clearance $(\mathrm{CrCl})<30 \mathrm{ml} / \mathrm{min}$ were also excluded.

Eligible patients were randomized to the rivaroxaban and warfarin treatment groups in accordance with the Japanese AF guidelines. Rivaroxaban was given orally once daily at a dose of $15 \mathrm{mg} /$ day, or $10 \mathrm{mg} /$ day for patients with $\mathrm{CrCl} 30-49 \mathrm{ml} / \mathrm{min}$ at randomization. Dose-adjusted warfarin was given at a target
INR of 2.0-3.0 and 1.6-2.6 to patients aged $<70$ years and those aged $\geq 70$ years, respectively. The pre-specified maximum exposure period was 30 months. At the end-of-study visit or at an earlier discontinuation visit, patients were transitioned from study medication to open-label warfarin or other appropriate therapy by the investigator in accordance with the clinical guideline. Follow-up was completed at the follow-up visit 30 days after the end-of-study visit or at the early discontinuation visit.

\section{Outcomes}

The principal safety outcomes were defined as a composite of major and non-major clinically relevant bleeding. The primary efficacy endpoint was defined as a composite of stroke and non-CNS systemic embolism.

An independent clinical endpoint committee adjudicated all suspected strokes, systemic embolisms, myocardial infarctions, deaths, and bleeding events contributing to the pre-specified endpoints.

\section{Time in Therapeutic Range (TTR)}

For each patient who received warfarin, individual TTR was based on regression analysis, calculated from INR using linear interpolation. The cumulative total of patient treatment days was the denominator, and the total number of days that INR was in the target range was the numerator.

\section{Statistical Analysis}

The primary objective of J-ROCKET AF was to test whether rivaroxaban was non-inferior to warfarin with respect to principal safety outcome in the safety group, which included all patients who received $\geq 1$ dose of the investigational drug, on 

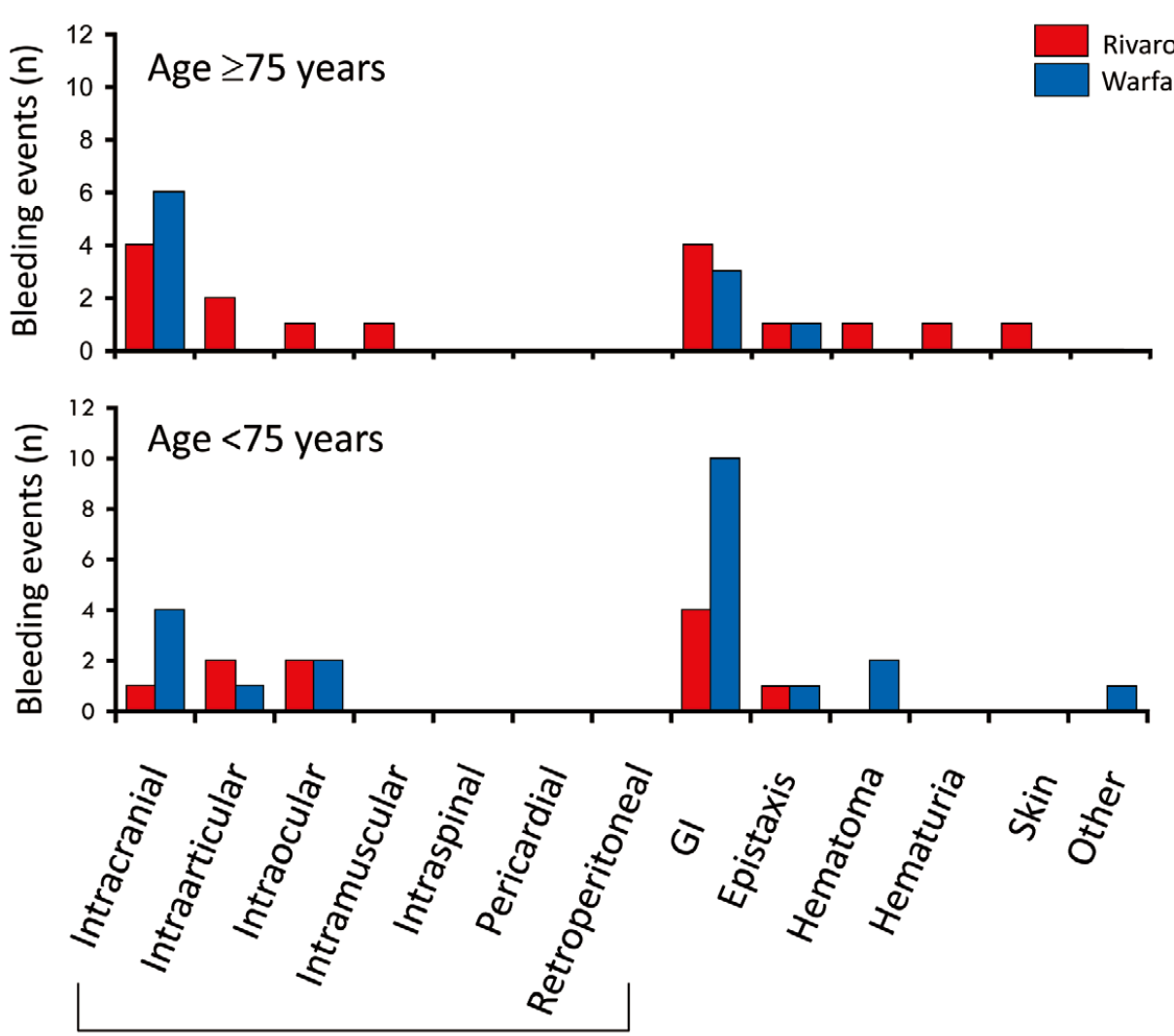

Critical Sites

Figure 3. Most frequent major bleeding site in patients aged $\geq 75$ years or younger. Safety group, on treatment. GI, gastrointestinal.

treatment, which was defined as the period from the first dose of study drug up to 2 days after the last dose, as evaluated on Cox proportional hazard model. The study was not intended to test efficacy hypotheses, but efficacy endpoints were evaluated on treatment, in the per protocol patients (patients in the safety group without any major protocol violations) in an exploratory manner.

The event rates (\% per year) of the principal safety outcome and primary efficacy endpoint were calculated by treatment group and by age $\geq 75$ years and $<75$ years. In addition, the hazard ratio (HR; rivaroxaban/warfarin) and $95 \%$ confidence interval $(\mathrm{CI})$ were calculated for the treatment group aged $\geq 75$ years and $<75$ years based on the Cox proportional hazard model that included the treatment group as a covariate. Two-sided $\mathrm{P}$-value for the interaction of the treatment group aged $\geq 75$ years and $<75$ years based on the Cox proportional hazard model, aging, and their interaction as covariates was provided. For the principal safety outcome, cumulative event rates were summarized using the Kaplan-Meier method.

\section{Results}

\section{Patient Characteristics}

Baseline characteristics according to age are listed in Table. The overall proportion of elderly patients (aged $\geq 75$ years) was $39.0 \%$. Patients aged $\geq 75$ years tended to have lower body weight, and approximately $40 \%$ had impaired renal function $(\mathrm{CrCl}<50 \mathrm{ml} / \mathrm{min})$. Their $\mathrm{CHADS}_{2}$ scores were $>3.0$, with the mean score being 3.6-3.7; many had complications of congestive heart failure and hypertension, and a few had complications of diabetes mellitus and prior stroke, transient ischemic attack, or non-CNS embolism.

\section{Safety Outcomes}

In elderly patients, the event rate (\%/year) of the principal safety outcome was 25.05 for the rivaroxaban group and 16.95 for the warfarin group, indicating a tendency to a higher event rate in the rivaroxaban group (Figures 1,2). In non-elderly patients, however, the event rate was 14.18 for the rivaroxaban group and 16.13 for the warfarin group, which showed a statistically significant interaction $(\mathrm{P}=0.04)$.

Sites of major bleeding are given in Figure 3. In elderly patients, intracranial hemorrhage ( $\mathrm{ICH}$ ) occurred in 4 patients on rivaroxaban and in 6 patients on warfarin, whereas in non-elderly patients, ICH occurred in 1 patient and in 4 patients, respectively.

\section{Efficacy Endpoints}

In elderly patients, the event rate of the primary efficacy endpoint was 2.18 for the rivaroxaban group and 4.25 for the warfarin group (HR, 0.51; 95\% CI: 0.20-1.27), whereas in nonelderly patients, the event rate was 0.72 for the rivaroxaban group and 1.67 for the warfarin group (HR, 0.44; 95\% CI: $0.13-1.42)$, with no statistically significant interaction $(\mathrm{P}=0.82)$. These results indicate that efficacy of rivaroxaban compared with warfarin was consistent irrespective of age (Figures 4,5). 


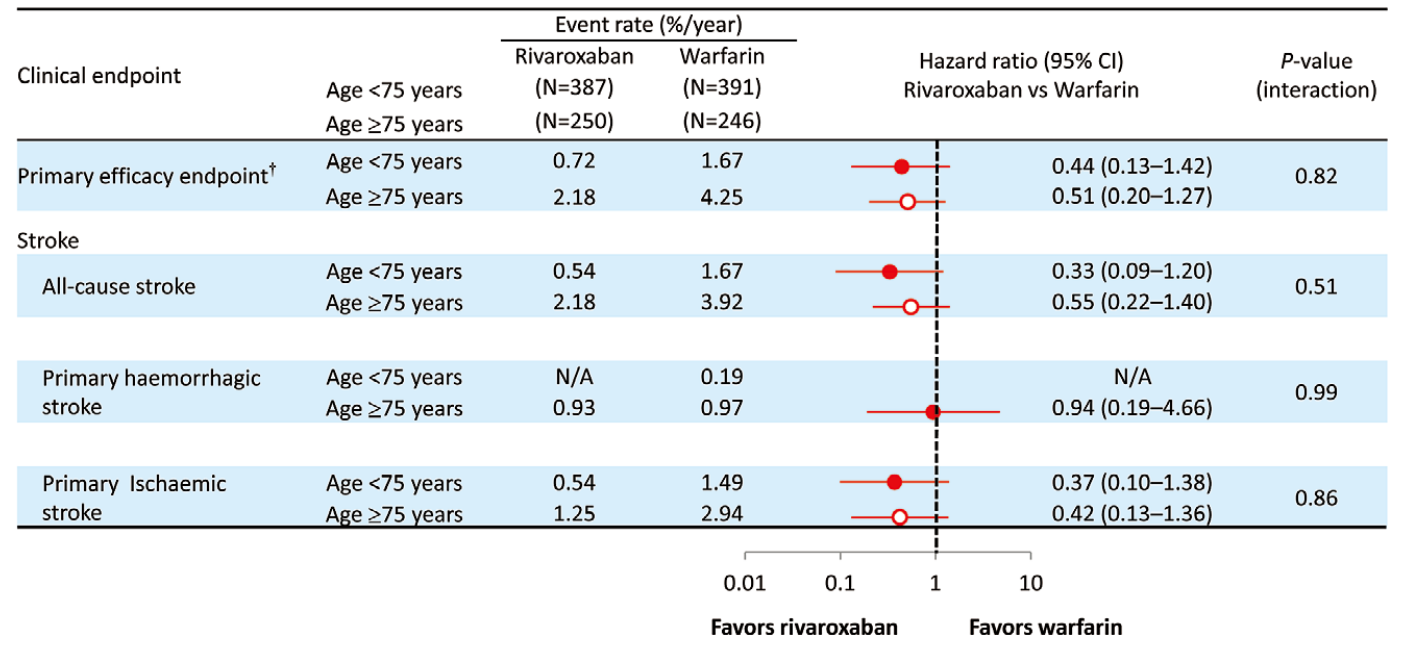

Figure 4. Primary efficacy endpoint according to age ( $\geq 75$ years or younger). Per protocol, on treatment. †Stroke plus non-central nervous system systemic embolism. $\mathrm{Cl}$, confidence interval; N/A, not applicable.

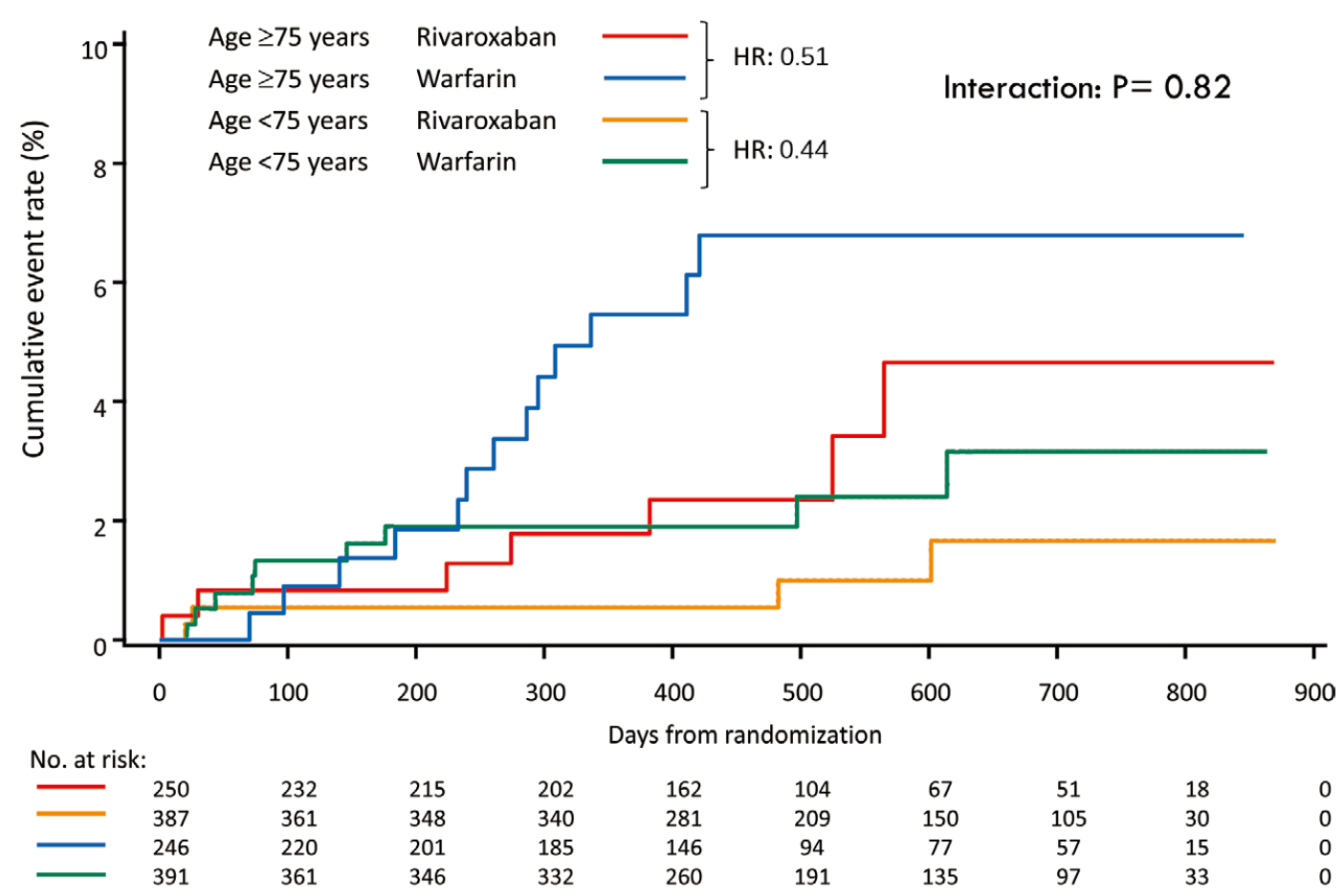

Figure 5. Kaplan-Meier curve of incidence of stroke or systemic embolism according to age ( $\geq 75$ years or younger). Per protocol, on treatment. HR, hazard ratio.

\section{Elderly Patients and Renal Function}

Further analysis by renal function in elderly patients with AF is shown in Figure 6. In patients with preserved renal function $(\mathrm{CrCl} \geq 50 \mathrm{ml} / \mathrm{min})$, the principal safety outcome occurred in $23.31 \%$ /year with rivaroxaban vs. $13.19 \%$ /year on warfarin (HR, 1.75; 95\% CI: 1.02-3.00). In those with moderate renal impairment, it occurred in $27.66 \%$ /year vs. $21.71 \%$ /year (HR,
1.30; 95\% CI: 0.77-2.20), respectively. No significant interaction was observed between those with preserved renal function and those with moderate renal impairment $(\mathrm{P}=0.43$ for interaction).

Furthermore, in patients with preserved renal function, the primary efficacy endpoint occurred in $1.03 \%$ /year with rivaroxaban vs. $4.22 \% / y e a r$ on warfarin (HR, 0.24 ; $95 \%$ CI: $0.05-$ 


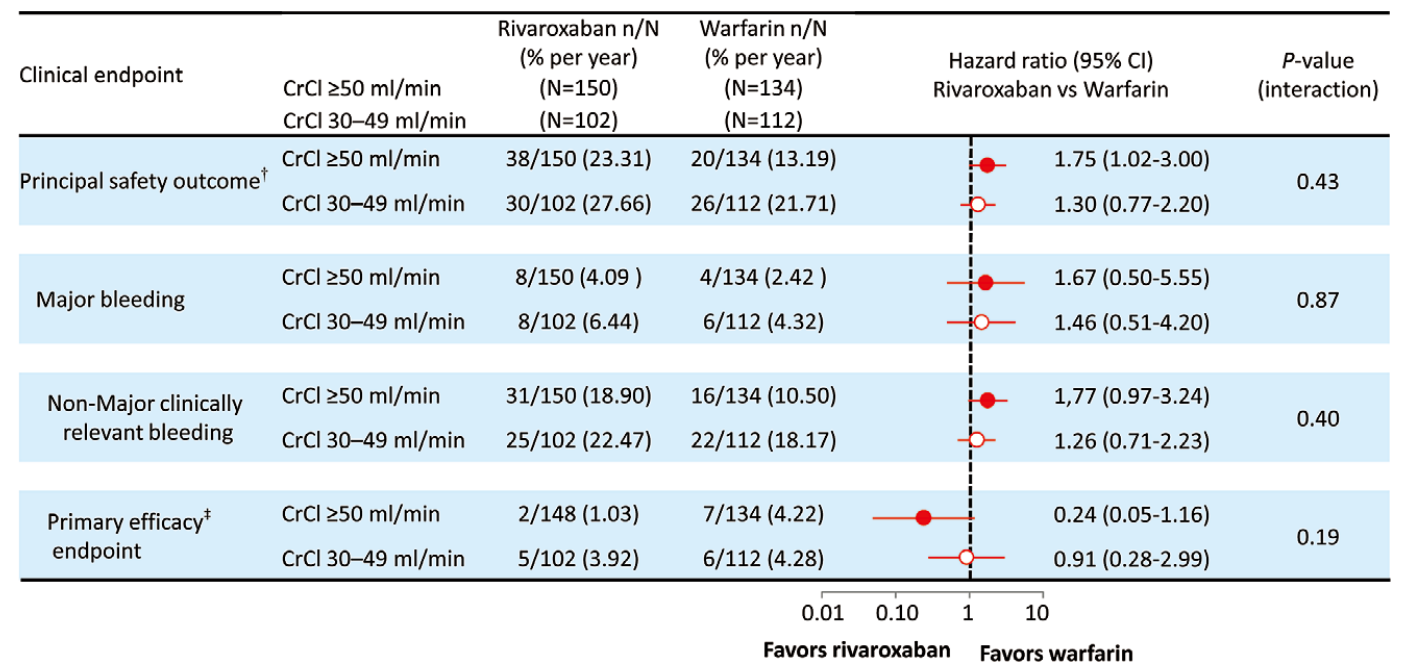

Figure 6. Safety and efficacy according to treatment group and renal function in patients aged $\geq 75$ years. ${ }^{\dagger}$ Major or non-major clinically relevant bleeding; ¥stroke or systemic embolism. $\mathrm{Cl}$, confidence interval; $\mathrm{CrCl}$, creatinine clearance.

1.16), and in $3.92 \% / y e a r$ on rivaroxaban vs. $4.28 \% /$ year on warfarin in those with moderate renal impairment (HR, 0.91; 95\% CI: 0.28-2.99). No significant interaction was observed between those with preserved renal function and those with moderate renal impairment $(\mathrm{P}=0.19$ for interaction).

\section{TTR}

For overall J-ROCKET AF, $65.0 \%$ of the INR of warfarintreated patients were within the pre-specified, age-dependent target range. For those aged $\geq 75$ years, $69.3 \%$ of values were within the therapeutic INR range, but for those aged $<75$ years only $57.3 \%$ of values were within this range (Table S1).

\section{Discussion}

The subgroup analysis of the J-ROCKET AF study by age has found that bleeding events rates were higher in the rivaroxaban group in patients aged $\geq 75$ years compared with the warfarin group. In contrast to the safety, the incidence rate of stroke or systemic embolism was lower in the rivaroxaban group compared with the warfarin group regardless of age, indicating that careful judgment is required for physicians with regard to the risk and benefit of rivaroxaban treatment in elderly patients.

\section{Net Clinical Benefit (NCB) of Anticoagulation in Elderly AF Patients}

The most important issue in the treatment of AF patients with anticoagulants is the optimization of the balance between efficacy and safety. Unfortunately, complications of bleeding in warfarin treatment are common in elderly patients. Particularly, $\mathrm{ICH}$ is a serious problem. Although the incidence rate of ICH is low, ${ }^{10}$ it has been reported that approximately $90 \%$ of warfarin-related deaths and disabilities in survivors are associated with $\mathrm{ICH}$, indicating that $\mathrm{ICH}$ is the most serious outcome. ${ }^{11}$ Singer et al advocated the NCB of anticoagulants by giving a weighting of 1.5 to ICH. ${ }^{12}$ They noted a positive NCB of warfarin in older patients with $\mathrm{AF}$, but not in younger ones, and also a positive NCB of warfarin in patients with AF with
$\mathrm{CHADS}_{2}$ scores $\geq 2 .{ }^{12}$ Aging is an important component of the $\mathrm{CHADS}_{2}$ score, and patients aged $\geq 75$ years inevitably have a $\mathrm{CHADS}_{2}$ score $\geq 1$. Moreover, in elderly patients, the number of patients with other complications such as hypertension and diabetes mellitus is higher. Also the mean $\mathrm{CHADS}_{2}$ score in the present patients aged $\geq 75$ years was 3.7 compared with 3.0 in those aged $<75$ years. Furthermore, in a subgroup analysis using the results of J-ROCKET AF, according to the NCB calculated by the method advocated by Singer et al, rivaroxaban was significantly favorable compared to warfarin in patients with $\mathrm{CHADS}_{2}$ score $\geq 2 .{ }^{13}$ Thus, rivaroxaban is considered more suitable for anticoagulant therapy in elderly patients with a high $\mathrm{CHADS}_{2}$ score compared with warfarin.

\section{Stroke Prevention With Anticoagulation in Elderly AF Patients}

Warfarin is the gold-standard for stroke prevention in patients with $\mathrm{AF}$. In a meta-analysis, warfarin was clearly shown to reduce the incidence rate of stroke in patients with AF by $64 \%$ relative to placebo or control. ${ }^{14}$ Despite this evidence, however, warfarin is underused and poorly managed, particularly in elderly patients, who carry the highest burden of disease owing to stroke. ${ }^{15}$ Several studies have suggested that warfarin is prescribed to only two-thirds of individuals with AF who are eligible for anticoagulant. ${ }^{16}$ In contrast, in the clinical setting in the J-RHYTHM Registry, the largest registry study in Japan, anticoagulant therapy was carried out in a large number of patients aged $\geq 70$ years, with $89.2 \%$ being prescribed warfarin. ${ }^{17-19}$ The reason for the higher prescription rate is the difference in the background of the recruited patients. The JRHYTHM registry recruited patients only from cardiovascular centers throughout Japan. In addition, with regard to the other clinical practice data in Japan, the Fushimi AF registry is a community-based survey of AF patients..$^{20}$ Compared with the J-RHYTHM registry, the warfarin prescription rate was much lower; $48.5 \%$ vs. $89 \%$, respectively. In the Fushimi AF registry there was a total of 76 institutions, a large proportion of which were private clinics of general practitioners. Therefore, 
as in the previous reports, ${ }^{16}$ the Fushimi AF registry may most likely reflect real clinical practice in Japan. These data suggested that the prescription rate of anticoagulants for patients with $\mathrm{AF}$ is still low. Another barrier to optimal warfarin therapy is poor management of INR in clinical practice. To maximize the benefit of warfarin treatment, Japanese AF guidelines recommend that patients aged $\geq 70$ years should receive warfarin with a target INR range of 1.6-2.6, or 2.0-3.0 for those aged $<70$ years. The primary analysis of J-ROCKET AF, however, showed that $72.7 \%$ of the INR in patients aged $<70$ years were within the range 1.6-2.6. ${ }^{9}$ In this subgroup analysis of the JROCKET AF study, patients aged $<75$ years had a lower TTR (eligible INR 2.0-3.0 for $<70$ years and 1.6-2.6 for $\geq 70$ years) of only $57.3 \%$ (Table S1). It has already been reported that the decrease in interpolated percentage TTR of INR increases the risk of stroke. ${ }^{21}$ In addition, the results of the EURO Heart Survey suggest that anti-thrombotic undertreatment of highrisk patients with AF was associated with a higher cardiovascular mortality during 1 year, whereas overtreatment was not associated with a higher risk for major bleeding. ${ }^{22}$ Despite this strong evidence for warfarin management, it seems that many physicians are too much concerned about the possible bleeding risk and tend to underestimate the potential benefits of warfarin therapy. ${ }^{23}$ Compared with warfarin, rivaroxaban is easier to use with the fixed dose regimen, without the need for monitoring for dose adjustment, and fewer interactions with concomitant drugs and food containing vitamin $\mathrm{K}$. It might be particular beneficial for clinicians to provide this therapeutic option for older patients.

\section{Renal Function in Elderly AF Patients}

Renal impairment is an independent risk factor for both stroke and bleeding in AF patients. ${ }^{24,25}$ Among elderly AF patients, renal impairment is more often observed than in the general population. Indeed, in this subgroup analysis, patients aged $\geq 75$ years had approximately more than 4-fold impaired renal function $(\mathrm{CrCl}<50 \mathrm{ml} / \mathrm{min}$; Table). Therefore, we further analyzed the safety and efficacy outcomes in elderly patients with renal impairment according to treatment. As shown in Figure 6, in elderly patients, the primary safety outcome occurred more frequently in those on rivaroxaban compared to warfarin regardless of renal function. In particular, in patients with preserved renal function, major bleeding events occurred in 8 patients on rivaroxaban and in 4 patients on warfarin. With regard to bleeding at critical sites, however, ICH occurred in 2 patients on rivaroxaban and in 3 patients on warfarin, and fatal bleeding occurred in 1 patient on warfarin. Although bleeding events occurred more frequently in patients on rivaroxaban, the number of critical bleeding events such as fatal bleeding or ICH was lower in the rivaroxaban arm compared with the warfarin arm. In contrast, in elderly patients with preserved renal function, patients on rivaroxaban had a marginally favorable trend in incidence rate of primary efficacy endpoint compared with patients on warfarin. One possible explanation for these differences is the intensity of warfarin treatment, which is a reduced target INR of $1.6-2.6$ in patients aged $\geq 70$ years according to the Japanese guideline. This might relatively affect the lower rate of bleeding events on warfarin than on rivaroxaban. From the aspect of efficacy, the potential effects of anticoagulant therapy for stroke prophylaxis might be insufficient in the lower intensity of warfarin treatment. Aging is known to be an independent risk factor not only for bleeding during anti-thrombotic therapy, but also for ischemic stroke and systemic embolism in AF patients. Because the bleeding event rate was higher in the rivaroxaban group in patients aged $\geq 75$ years compared to the warfarin group, treatment would need to be personalized based on the risk and benefit of anticoagulant therapy, especially for elderly patients with AF.

\section{Study Limitations}

J-ROCKET AF was not sufficiently powered to evaluate efficacy due to the limited number of patients. This trial was also not primarily designed to detect interactions between study drug and patient age. Thus, the present data should be interpreted with caution because the number of patients is limited for further subgroup analyses, such as between study drug and renal function in the younger or older subgroups. Another limitation is that, in this subgroup analysis, patients aged $\geq 75$ years tended to have lower body weight and higher $\mathrm{CHADS}_{2}$ score, and approximately $40 \%$ had impaired renal function $(\mathrm{CrCl}$ $<50 \mathrm{ml} / \mathrm{min}$ ). The proportion of women was higher in the patients aged $\geq 75$ years. Also, the concomitant disorders varied greatly between elderly patients and non-elderly patients. Therefore, it should be noted that these co-founding factors other than age may affect safety and efficacy in this subgroup analysis. Furthermore, the present results were derived from a selected patient group for the clinical trial, which may deviate from an unselected patient population in the real world.

\section{Acknowledgments}

The rivaroxaban clinical development program is co-sponsored by Janssen Pharmaceuticals (Raritan, NJ, USA) and Bayer HealthCare Pharmaceuticals (Leverkusen, Germany). The trial was funded by Bayer HealthCare Pharmaceutical's Japanese subsidiary, Bayer Yakuhin. Dr Hori has received consultancy fees from Bayer, Boehringer Ingelheim, Bristol MyersSquibb, and Pfizer. Dr Matsumoto and Dr Momomura have received consultancy fees from Bayer. Dr Tanahashi has received consultancy fees from Bayer and Mitsubishi Tanabe, and honoraria from Mitsubishi Tanabe and Sanofi-Aventis. Dr Uchiyama has received consultancy fees from Bayer and Boehringer Ingelheim and research grants from Bayer, Boehringer Ingelheim, and Daiichi-Sankyo. Dr Goto has received research grants from Astellas, AstraZeneca, Daiichi, Eisai, Kowa, Ono, Otsuka, Pfizer, Sanofi-Aventis, and Takeda, and honoraria from Daiichi-Sankyo, Eisai, Otsuka, Sanofi-Aventis, and Schering-Plough. Dr Izumi has received consultancy fees from Bayer and Pfizer. Dr Koretsune has received honoraria from Bayer, Boehringer Ingelheim, Daiichi-Sankyo, and Bristol MyersSquibb. Drs Iekushi, Yamanaka, Kajikawa, and Ueda, and Mr Tajiri and $\mathrm{Mr}$ Kato report employment by Bayer Yakuhin. No other conflicts of interest are reported.

\section{References}

1. Wolf PA, Abbott RD, Kannel WB. Atrial fibrillation: A major contributor to stroke in the elderly: The Framingham Study. Arch Intern Med 1987; 147: 1561-1564.

2. Lip GY, Tse HF, Lane DA. Atrial fibrillation. Lancet 2012; 379: 648-661.

3. Lip GY, Lane DA. Stroke prevention with oral anticoagulation therapy in patients with atrial fibrillation: Focus on the elderly. Circ J 2013; 77: $1380-1388$.

4. Gage BF, Waterman AD, Shannon W, Boechler M, Rich MW, Radford MJ. Validation of clinical classification schemes for predicting stroke: Results from the National Registry of Atrial Fibrillation. JAMA 2001; 285: 2864-2870.

5. Pisters R, Lane DA, Nieuwlaat R, de Vos CB, Crijns HJ, Lip GY. A novel user-friendly score (HAS-BLED) to assess 1-year risk of major bleeding in patients with atrial fibrillation: The Euro Heart Survey. Chest 2010; 138: $1093-1100$.

6. Kubitza D, Becka M, Roth A, Mueck W. Dose-escalation study of the pharmacokinetics and pharmacodynamics of rivaroxaban in healthy elderly subjects. Curr Med Res Opin 2008; 24: 2757-2765.

7. Kubitza D, Becka M, Wensing G, Voith B, Zuehlsdorf M. Safety, pharmacodynamics, and pharmacokinetics of BAY 59-7939 - an oral, direct Factor Xa inhibitor - after multiple dosing in healthy male subjects. Eur J Clin Pharmacol 2005; 61: 873-880.

8. Perzborn E, Strassburger J, Wilmen A, Pohlmann J, Roehrig S, Schlemmer $\mathrm{KH}$, et al. In vitro and in vivo studies of the novel antithrombotic agent BAY 59-7939: An oral, direct Factor Xa inhibitor. J Thromb Haemost 2005; 3: 514-521. 
9. Hori M, Matsumoto M, Tanahashi N, Momomura S, Uchiyama S, Goto S, et al. Rivaroxaban vs. warfarin in Japanese patients with atrial fibrillation: The J-ROCKET AF study. Circ J 2012; 76: $2104-2111$

10. Mant J, Hobbs FD, Fletcher K, Roalfe A, Fitzmaurice D, Lip GY, et al. Warfarin versus aspirin for stroke prevention in an elderly community population with atrial fibrillation (the Birmingham Atrial Fibrillation Treatment of the Aged Study, BAFTA): A randomised controlled trial. Lancet 2007; 370: 493-503.

11. Fang MC, Go AS, Chang Y, Hylek EM, Henault LE, Jensvold NG, et al. Death and disability from warfarin-associated intracranial and extracranial hemorrhages. Am J Med 2007; 120: 700-705.

12. Singer DE, Chang Y, Fang MC, Borowsky LH, Pomernacki NK, Udaltsova N, et al. The net clinical benefit of warfarin anticoagulation in atrial fibrillation. Ann Intern Med 2009; 151: 297-305.

13. Hankey GJ, Patel MR, Stevens SR, Becker RC, Breithardt G, Carolei A, et al. Rivaroxaban compared with warfarin in patients with atrial fibrillation and previous stroke or transient ischaemic attack: A subgroup analysis of ROCKET AF. Lancet Neurol 2012; 11: 315-322.

14. Hart RG, Pearce LA, Aguilar MI. Meta-analysis: Antithrombotic therapy to prevent stroke in patients who have nonvalvular atrial fibrillation. Ann Intern Med 2007; 146: 857-867.

15. Sinnaeve PR, Brueckmann M, Clemens A, Oldgren J, Eikelboom J, Healey JS. Stroke prevention in elderly patients with atrial fibrillation: Challenges for anticoagulation. J Intern Med 2012; 271: 15-24.

16. Nieuwlaat R, Capucci A, Camm AJ, Olsson SB, Andresen D, Davies $\mathrm{DW}$, et al. Atrial fibrillation management: A prospective survey in ESC member countries: The Euro Heart Survey on Atrial Fibrillation. Eur Heart J 2005; 26: 2422-2434.

17. Atarashi H, Inoue H, Okumura K, Yamashita T, Kumagai N, Origasa $\mathrm{H}$, et al. Present status of anticoagulation treatment in Japanese patients with atrial fibrillation: A report from the J-RHYTHM Registry. Circ J 2011; 75: 1328-1333.

18. Atarashi $\mathrm{H}$, Inoue $\mathrm{H}$, Okumura $\mathrm{K}$, Yamashita $\mathrm{T}$, Origasa $\mathrm{H}, \mathrm{J}-$ RHYTHM Registry Investigators. Investigation of optimal anticoagulation strategy for stroke prevention in Japanese patients with atrial fibrillation: The J-RHYTHM Registry study design. J Cardiol 2011; 57: 95-99.
19. Inoue $\mathrm{H}$, Okumura $\mathrm{K}$, Atarashi $\mathrm{H}$, Yamashita T, Origasa $\mathrm{H}$, Kumagai $\mathrm{N}$, et al. Target international normalized ratio values for preventing thromboembolic and hemorrhagic events in Japanese patients with non-valvular atrial fibrillation. Circ J 2013; 77: 2264-2270.

20. Akao M, Chun YH, Wada H, Esato M, Hashimoto T, Abe M, et al. Current status of clinical background of patients with atrial fibrillation in a community-based survey: The Fushimi AF Registry. J Cardiol 2013; 61: 260-266.

21. Ageno W, Gallus AS, Wittkowsky A, Crowther M, Hylek EM, Palareti G, et al. Oral anticoagulant therapy: Antithrombotic therapy and prevention of thrombosis, 9th edn: American College of Chest Physicians Evidence-Based Clinical Practice Guidelines. Chest 2012; 141(2 Suppl): e44S-e88S, doi:10.1378/chest.11-2292.

22. Nieuwlaat R, Olsson SB, Lip GY, Camm AJ, Breithardt G, Capucci A, et al. Guideline-adherent antithrombotic treatment is associated with improved outcomes compared with undertreatment in high-risk patients with atrial fibrillation. Am Heart J 2007; 153: 1006-1012.

23. Man-Son-Hing M, Laupacis A. Anticoagulant-related bleeding in older persons with atrial fibrillation: Physicians' fears often unfounded. Arch Intern Med 2003; 163: 1580-1586.

24. Go AS, Fang MC, Udaltsova N, Chang Y, Pomernacki NK, Borowsky $\mathrm{L}$, et al. Impact of proteinuria and glomerular filtration rate on risk of thromboembolism in atrial fibrillation: The anticoagulation and risk factors in atrial fibrillation (ATRIA) study. Circulation 2009; 119: $1363-1369$.

25. Friberg L, Rosenqvist M, Lip GY. Evaluation of risk stratification schemes for ischaemic stroke and bleeding in 182678 patients with atrial fibrillation: The Swedish Atrial Fibrillation cohort study. Eur Heart J 2012; 33: 1500-1510.

\section{Supplementary Files}

Supplementary File 1

Table S1. Proportion of INR within therapeutic target range in the warfarin group

Please find supplementary file(s);

http://dx.doi.org/10.1253/circj.CJ-13-1324 